\title{
Nanoscale nights of COVID-19
}

\author{
As the spread of SARS-CoV-2 has triggered worldwide closures of research labs and facilities, Kostas Kostarelos \\ shares his views on what may be going wrong in the fight against COVID-19 and how the nanoscience community \\ could and should contribute.
}

\section{The beauty of viral nanoparticles}

During several years of teaching nanomedicine, I have illustrated the uniqueness of viral nanoparticles to many generations of students: "Remember, viruses are the most beautiful, smart and capable nanoparticles!" I have always enthusiastically highlighted their structurally beautiful nanoscale features, and their biological trickery in transferring their genetic contents into target cells and hijacking them to express proteins. Viruses are evolutionary works of art and in the fields of gene therapy, vaccinology and immunotherapy (to name just a few), scientists are engineering and using viruses for the smart delivery of molecules and genetic information. However, I must admit that during the past few late nights, while working in my makeshift home office, I cannot help but feel strangely guilty for my past opinions and lecture notes. As I gradually get more gloomy looking at the daily updated counts of 'confirmed cases', 'deaths' and 'recovered cases', guilt starts taking over. How can such beautiful nanoparticles create so much human loss, havoc and devastation? How can such nanoscale beauty so rapidly metamorphose into a wild beast?

\section{The human factor}

We knew this was coming. Experts and international committees have long been analysing and developing scenarios and plans to be able to respond to a potential intentional or unintended virus outbreak. There were also warnings that owing to our excessively industrialized agricultural and animal farming activities, climate change, highly congested urban areas and international aviation, which has created a 'smaller' and more accessible world, the risk for the spread of a contagious virus or an antibiotic-resistant bacterial strain was almost certain to happen. Not only were we told, but we had been given warning signs throughout the past 20 years, including avian influenza virus (Hong Kong, 1997), severe acute respiratory syndrome (SARS) coronavirus (China, 2002), swine influenza virus (Mexico, 2009) and Middle East respiratory syndrome (MERS) coronavirus (Saudi Arabia, 2012). These magnificent nanostructures that combine structural complexity with biological efficiency

(Fig. 1), have all been originally transmitted from different animal species to humans; importantly, they are highly contagious with human-to-human transmission, which has sadly caused many deaths. Unfortunately, we collectively failed to understand that the risk was clear, imminent and significant. We failed to guarantee that the plans and directives, developed by experts, can be rapidly and effectively implemented in case of an outbreak. Indeed, each efficient viral infection is met by a multiplicity of human inefficiencies. We were out-beaten by nature's nanostructures.

\section{The scientific facts}

The acute need is to get the scientific facts right, as fast and accurately as possible. We need to take advantage of the technology and automation in our laboratories as well as powerful modelling approaches to reveal scientific facts about COVID-19. What have we already learnt? SARS-CoV-2 is very similar to previously studied coronaviruses. It binds to angiotensin I converting enzyme 2 (ACE2) cell receptors and is highly transmittable among humans, even at the latent stages of infection. We know that SARS-CoV-2 infects the upper respiratory system and, in particular, the lung epithelium, with a severe downstream effect or direct impact on the myocardium. Infections of older (male more than female) individuals, and of patients with chronic underlying (diagnosed or not) conditions are the most vulnerable. Lastly, we know that $80 \%$ of infected individuals are asymptomatic or suffer from mild symptoms, $15 \%$ need hospitalization and 5\% develop a serious illness and may need critical care. We also know though, that valid epidemiological data should be taken with great caution as different countries and health systems have very different approaches to testing and reporting.

However, there is also a lot we do not know. We do not know whether SARS-CoV-2 is able to mutate into different serotypes and how rapidly. We do not know whether cell internalization and viral transduction of SARS-CoV-2 is dependent on temperature, multiple rounds
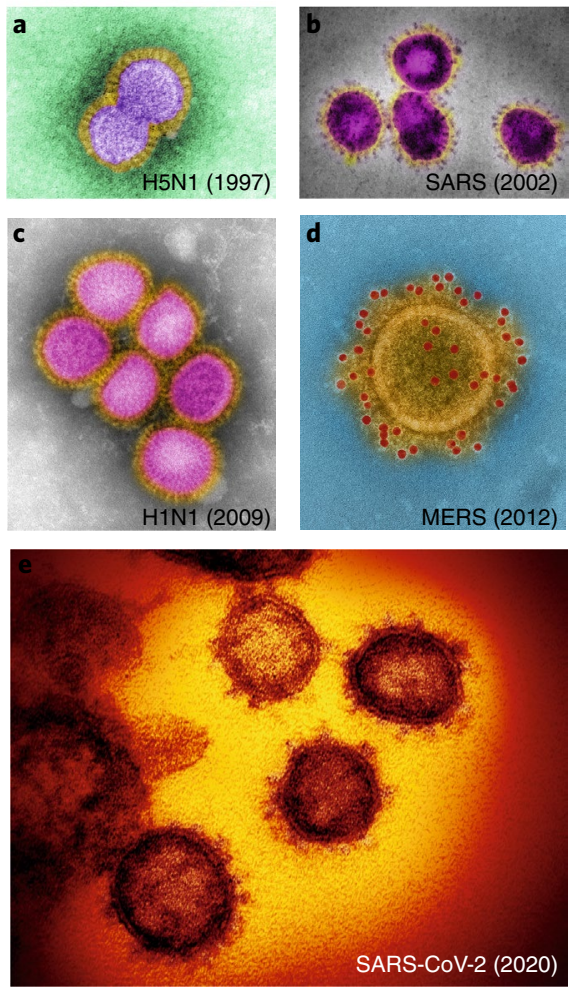

Fig. 1 | Electron micrographs of virus structures. a-e, False coloured images of avian influenza virus (a), SARS coronavirus (b), swine influenza virus (c), MERS coronavirus (d) and SARS-CoV-2 (e). Credit: Science Source (a); Getty/Callista Images $(b, c)$; NIAID (d); NIAID-RML (e)

of infections and/or generations of virus progeny. We do not know whether the antibodies and titres produced in infected individuals are able to confer effective immunity against subsequent exposures to SARS-CoV-2 and for how long such immunity may last. Finally, we need to agree on strict parameters for the clinical reporting of COVID-19 infections and outcomes, which all national health agencies should adhere to. This is the only way to determine valid transmission, fatality and protective immunity rates. Only if we generate accurate scientific knowledge (even if inconclusive), can we assess the validity of public health interventions that are currently in place in many countries around the 
world, and potentially reduce the demand for such dramatic societal sacrifices.

\section{Where have all the (nano)scientists gone?}

In order to generate the urgently needed scientific knowledge, we need to get our technically capable, bright, young scientists back in the labs. I cannot help but wonder, why most governments in Europe (and more recently also in the USA) ordered the closures of world-class research laboratories and their support systems, during a generational crisis of biological and biomedical emergency. Many trained biological or biomedical scientists have been sent home, their laboratories closed down and their reagents left to expire. Of course, scientists would have to avoid working in confined spaces, should wear protective gear and would have to be regularly monitored for infection - but we need our laboratories back. Our energies and efforts should be focused on how to contribute to the fight against COVID-19. We - the biomedical scientific community - have the responsibility to support our clinical and healthcare colleagues, who work on the frontline of the pandemic, with our technical and intellectual capabilities in generating relevant and much needed scientific knowledge. We need to maximize synergies free from any political, linguistic, financial, geographical or scientific discipline obstacles. It is shameful that highly trained scientists are considered 'non-essential', while our nurses and clinical colleagues are daily exposed to the virus in their efforts to treat a constantly increasing number of patients.

\section{The cancer analogy}

Many heated conversations are currently taking place as to the best way to manage the COVID-19 menace. As I have tried to follow the different opinions, I cannot stop thinking of an analogy to cancer nanotechnology, that is, the three key principles in managing an individual cancer patient: early detection, monitoring and targeting. These principles, if exercised simultaneously, could also be illuminating as a way forward in the management of the COVID-19 pandemic. Early detection has improved the prognosis of many cancer patients. Similarly, early detection of individuals and groups, who are infected with COVID-19, could substantially accelerate the ability to manage and treat patients, but also infection hotspots. All chronic conditions, such as cancer, are further managed by regular monitoring. Therefore, monitoring should be undertaken not only for patients already infected with COVID-19, to track progression and responses, but also for healthy essential workers to ensure that they remain healthy and to reduce the risk of further spreading. Finally, nanomaterials (as well as other biologicals, such as monoclonal antibodies) are often used for targeting therapeutic agents specifically to cancer cells to minimize damage of healthy tissue.
The same principle of targeting should be applied in the management of COVID-19 to be able to effectively isolate and treat infected patients. But also to establish a 'protective targeting' strategy to shield the vulnerable segment of the population (by isolating them or by social distancing, but with provision of emotional and practical support), and protect 'essential' workers (by making sure that protective gear and monitoring is provided). Only if all three principles are applied, the rest of society can and should return to normal function to support the activities in managing the pandemic. In this way, I want to finish by paraphrasing an undergraduate cancer management textbook: The COVID-19 pandemic will become a chronic disease, and as for any other chronic medical condition, COVID-19 stricken societies have families, jobs, businesses and other commitments. Therefore, our aim is to cure COVID-19 if possible; however, if not curable, we need to control the symptoms to improve the quality of patients' lives by assuring society's function to be able to support its ill and vulnerable.

\section{Kostas Kostarelos (D) 1,2凶}

${ }^{1}$ Nanomedicine Lab, The University of Manchester, Manchester, UK. ${ }^{2}$ Catalan Institute of Nanoscience and Nanotechnology (ICN2), Barcelona, Spain.

$\bigotimes_{\text {e-mail: kostas.kostarelos@manchester.ac.uk }}$

Published online: 27 April 2020 https://doi.org/10.1038/s41565-020-0687-4 